\title{
ENETS Consensus Guidelines for the Standards of Care in Neuroendocrine Tumors: Towards a Standardized Approach to the Diagnosis of Gastroenteropancreatic Neuroendocrine Tumors and Their Prognostic Stratification
}

\author{
Günter Klöppel $^{\mathrm{a}}$ Anne Couvelard ${ }^{\mathrm{b}}$ Aurel Perren ${ }^{\mathrm{c}}$ Paul Komminoth $^{\mathrm{d}}$ Anne-Marie McNicole \\ Ola Nilsson ${ }^{f}$ Aldo Scarpag Jean-Yves Scoazech Bertram Wiedenmann ${ }^{\mathrm{i}}$ Mauro Papottij \\ Guido Rindik Ursula Plöckinger ${ }^{i}$ and all other Mallorca Consensus Conference participants \\ anstitut für Allgemeine Pathologie und Pathologische Anatomie, Universität Kiel, Kiel, Germany; \\ bService de Gastroentérologie, Hôpital Beaujon, Clichy, France; Institut für Allgemeine Pathologie und \\ Pathologische Anatomie der Technischen Universität München, Klinikum r.d. Isar, Munich, Germany; \\ dInstitute for Pathology, Stadtspital Triemli, Zürich, Switzerland; eDivision of Cancer Sciences and Molecular \\ Pathology, Pathology Department, Royal Infirmary, Glasgow, UK; 'Department of Pathology, Sahlgrenska Sjukhuset,

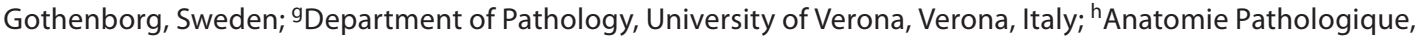 \\ Hôpital Edouard Herriot, Lyon, France; 'Department of Hepatology and Gastroenterology, Campus Virchow-Klinikum, \\ Charité-Universitätsmedizin Berlin, Berlin, Germany; jDepartment of Biological and Clinical Sciences, \\ University of Turin, St. Luigi Hospital, Turin, and kDipartimento di Patologia e Medicina di Laboratorio, \\ Sezione di Anatomia Patologica, Università degli Studi, Parma, Italy
}

\section{Introduction}

In order to assure an optimal treatment of gastroenteropancreatic neuroendocrine tumors (GEP-NETs), a standardized diagnostic procedure is required. This need prompted the European Neuroendocrine Tumor Society (ENETS) to organize a third Consensus Conference, which was held in La Palma (Mallorca) in 2007. One result was a proposal for a standardized approach to the morphological diagnosis of GEP-NETs, whose needs and options were designed, discussed and consensually approved. In addition, a working formulation for a diagnostic and prognostic stratification was proposed based on histological typing, differentiation, grading and TNM staging [1-3].

\section{KARGER}

Fax +4161306 1234

E-Mail karger@karger.ch

www.karger.com
(C) 2008 S. Karger AG, Basel

$0028-3835 / 09 / 0902-0162 \$ 26.00 / 0$

Accessible online at:

www.karger.com/nen

\section{Background}

Several recent publications have focused on the application of the WHO classification of GEP-NETs and proved its effectiveness, supporting the concept that the various GEP-NET types also differ in their clinical behavior. Because of this biological diversity, the treatment of GEP-NETs is becoming more and more type-specific [4-11].

Recent data from the German NET registry have revealed that approximately one third of the cases reported between 2000 and 2006 lack a documented histopathological diagnosis, and in even more cases the tentative diagnosis of a NET was not confirmed by using neuroendocrine markers, nor was the proliferation determined by using Ki67/MIB1 [Plöckinger, unpubl. data]. 
This emphasizes the need for a standardized morphological procedure that would result in a diagnosis sufficient to meet all criteria necessary for exact recognition and optimal treatment. During the recent Standards of Care Conference of ENETS held in La Palma (Mallorca, Spain) from November 29 to December 1, 2007, a protocol for the morphological diagnosis of GEP-NETs was discussed and approved. Here we report on the recommended diagnostic procedure, focusing on the needs and options.

\section{Needs and Options}

Tissue from GEP-NETs for use in diagnostic procedures is obtained either by biopsy from a primary tumor or a metastasis (i.e. liver, lymph node) or by surgical removal of tumor tissue. The biopsy specimen is usually formalin-fixed when it is received by the pathology laboratory, while the surgical resection specimen may arrive as fresh tissue that can be frozen and stored before the remaining tissue is fixed in formalin. The biopsy specimen is immediately processed for histopathological assessment, whereas the resection specimen requires a thorough and detailed gross examination and description of the lesions observed, before tissue blocks are sampled and obtained for histology. Gross examination and description are necessary in order to provide the data and criteria on which the TNM classification is based. In particular, information has to be provided on the resection margins, the tumor location, the relationship of the tumor to other organs, the tumor's size, invasiveness and composition and the presence of regional metastases.

\section{Diagnostic Standards}

Table 1 depicts the standard procedure for the diagnosis of NET in a biopsy specimen. It shows the 'musts and options' that follow the recognition of a tumor with endocrine features on an HE-stained section from a biopsy specimen. Crucial for the diagnosis is the demonstration of the neuroendocrine markers synaptophysin and chromogranin A in the tumor cells $[12,13]$. Synaptophysin, which is an integral membrane protein of small clear vesicles (diameter $40-80 \mathrm{~nm}$ ) occurring in all normal and neoplastic neuroendocrine cells, is diffusely expressed in the cytoplasm of all cells of a NET. Chromogranin A, which is a protein located in the matrix of large secretory granules $(>80 \mathrm{~nm})$ is, in contrast to synapto-
Table 1. Musts and options for assessing a biopsy specimen containing a tumor with features of a well- or poorly-differentiated GEP-NET

Must
Immunostaining for neuroendocrine markers
- Synaptophysin and chromogranin
Immunostaining for proliferation marker
- Ki67/MIB1
Optional
Immunostaining for hormones such as insulin, gastrin,
serotonin and others
Because of hormonal symptoms, liver metastases of
an unknown primary or follow-up of a tumor with a
hormonal syndrome
Immunostaining for somatostatin receptor (i.e. SSTR2)
Because of diagnostic/therapeutical tumor management
Immunostaining for vessel markers angioinvasion
Because of angioinvasion

physin, inhomogeneously expressed in the cytoplasm of the tumor cell or can even be lacking, since its expression depends on the number of neurosecretory granules present in the cells and on the cell type. In small cell neuroendocrine carcinoma of the lung (because of the small number of secretory granules per cell) and in rectal NETs (because of the specific cell type of these neoplasms), chromogranin A is usually lacking, but in most well-differentiated neuroendocrine neoplasms with abundant secretory granules, chromogranin A is intensely expressed. There is no need for a semiquantitative evaluation of synaptophysin and chromogranin A stainings, since such information is currently of no use for clinical purposes.

The application of neuron-specific enolase (NSE) and other markers such as CD56 (i.e. NCAM) and PGP9.5 for the recognition of GEP-NETs is discouraged, due to the frequent unspecificity of the available antibodies (in the case of NSE) or the unspecificity of the marker itself (in the case of CD56) [13]. For visualization of the suggested markers, staining techniques and antibodies that have been approved in quality assurance procedures should be used.

Once the neuroendocrine nature of the tumor has been established, its differentiation and proliferative activity have to be determined. As to the differentiation, well- and poorly-differentiated NETs have to be distinguished according to the WHO classification [14]. The proliferative activity can be determined by counting the mitoses per high-power field and/or - more easily - by 
Table 2. Grading proposal for GEP-NETs from Rindi et al. [1]

\begin{tabular}{lll}
\hline Grade & Mitotic count, $10 \mathrm{HPF}^{1}$ & Ki67 index, $\%^{2}$ \\
\hline G1 & 1 & $\leq 5$ \\
G2 & $2-20$ & $3-20$ \\
G3 & $>20$ & $>20$
\end{tabular}

${ }^{1} \mathrm{HPF}=$ high-power field $=2 \mathrm{~cm}^{2}$, at least 40 fields evaluated in areas at highest mitotic density.

${ }^{2}$ MIB1 antibody; \% of 2,000 cells in areas of highest nuclear labeling.

Table 3. Musts and options for the assessment of resected GEPNETs

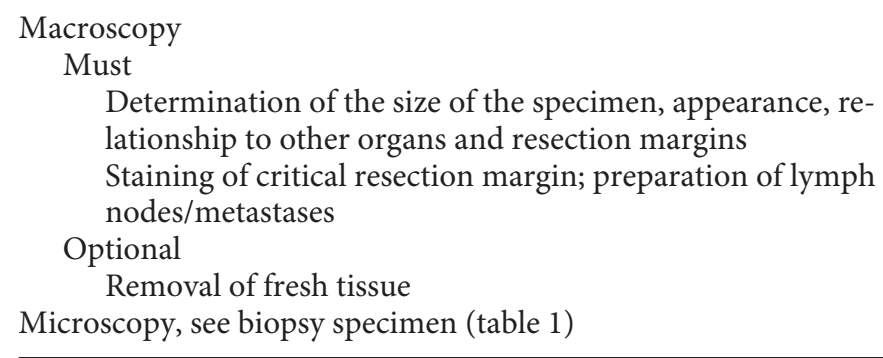

immunostaining for the cell cycle-dependent marker Ki67 (MIB1) antigen, which is expressed in the nucleus. As an alternative to Ki67, the proliferation cell nuclear antigen can be used [15]. To determine the Ki67 (MIB1) labeling index, 100 tumor cells have to be assessed in a hot-spot area. In case the Ki67 positivity is unevenly distributed, several tumor areas should be evaluated. The Ki67 labeling index then serves as the basis for grading the tumors as G1 (<2\%), G2 (>2-20\%) or G3 (>20\%) (table 2) $[1,2]$. G1 and G2 GEP-NETs are well differentiated and display diffuse and intense expression of the two neuroendocrine markers, chromogranin A and synaptophysin. G3 indicates a poorly differentiated neuroendocrine carcinoma that shows staining for synaptophysin but not (or only little) for chromogranin A.

Immunostaining for hormones that are specific to certain cell types in the intestine and the pancreas and are known to also occur in tumors is optional for the diagnosis of GEP-NETs. One reason for immunostaining is to verify the production of a hormone causing a syndrome at the tumor cell level [3]. Another reason is to identify a special tumor type, for instance a duodenal gangliocytic paraganglioma, by identifying hormones characterizing the neoplasm. The third reason is to identify hormones in a liver or lymph node biopsy specimen that shows a metastasis of a well-differentiated NET. The expression of certain hormones such as serotonin, gastrin, glucagon and pancreatic polypeptide may provide clues to the site of the primary tumor. Serotonin positivity suggests, first of all, a primary in the ileum; gastrin suggests a primary in the duodenum or the pancreas, and glucagon/pancreatic polypeptide suggests a primary in the pancreas.

Optional in the standardized protocol is also the application of antibodies for the immunostaining of somatostatin receptors, in particular of somatostatin receptor 2 (SSTR2). GEP-NETs that are positive for SSTR2 are, in the majority of cases, also depicted by an octreotide scan [16]. In some case, immunostaining for vessel markers to verify tumor angioinvasion may be helpful.

If the diagnosis is to be made on a resection specimen, the microscopic procedure is preceded by careful macroscopic examination (table 3). This results in data on the precise localization of the tumor, its size, its composition (presence of necrosis or cystic changes), its relationship to anatomic structures, resection margins and adjacent organs, and the presence of lymph node and other metastases. All of this information is needed in order to stage the tumor according to the recently proposed TNM staging classifications $[1,2]$. The tissue must be adequately sampled to allow a precise microscopic assessment of the tumor tissue. Formalin is recommended for the fixation of the tissue. The removal of fresh tissue for special biological examinations is optional.

The microscopic evaluation of the resected tumor follows the guidelines given above for tumor tissue in biopsy specimens. In addition, information has to be provided on invasion of lymph and blood vessels, nerves and resection margins. Staining for hormones is optional (table 1).

\section{Problems}

Gastrointestinal and pancreatic adenocarcinomas with mixed differentiation present a special challenge in the classification [17]. In most of these neoplasms, the cells staining for synaptophysin and chromogranin A represent a minor component of the total tumor cell population. Regarding their prognosis and treatment, it has been found that they behave like the respective adenocarcinomas without endocrine cells. The mixed carcinomas should therefore be clearly distinguished from the pure GEP-NETs. 
Table 4. Proposal for the stratification of GEP-NETs into three treatment groups based on growth features, TNM stages and grade

\begin{tabular}{|c|c|c|c|c|}
\hline \multicolumn{5}{|l|}{ Localized tumor } \\
\hline Very low risk of metastasis & Well-differentiated & G1 & $\mathrm{T} 1$ & Endoscopic resection \\
\hline Low risk & Well-differentiated & G1 & $\mathrm{T} 2$ & Surgery \\
\hline Intermediate risk & Well-differentiated & G2 & $\mathrm{T} 1$ & Surgery \\
\hline High risk & Well-differentiated & $\mathrm{G} 1 / 2$ & $\mathrm{~T} 2$ & Surgery \\
\hline Slow growth & Well-differentiated & G1 & $\mathrm{T} 1 / 2 / 3 \mathrm{~N} 1$ & Surgery \\
\hline Intermediate growth & Well-differentiated & G2 & $\mathrm{T} 1 / 2 / 3 \mathrm{~N} 1$ & Surgery, AT \\
\hline Fast growth & Poorly differentiated & G3 & $\mathrm{T} 1 / 2 / 3 \mathrm{~N} 1$ & Surgery, AT \\
\hline \multicolumn{5}{|c|}{ Nodal and hematogenous metastases } \\
\hline Slow growth & Well-differentiated & G1 & Any T N1M1 & Surgery, AT \\
\hline Intermediate growth & Well-differentiated & $\mathrm{G} 2$ & Any T N1M1 & Surgery, AT \\
\hline
\end{tabular}

AT $=$ Additional treatment, including biotherapy and/or chemotherapy.

\section{Prognostic Stratification}

On the basis of the information and data obtained from the macroscopic and microscopic evaluation, i.e. TNM stage, grade and tumor type, a prognostic stratification of the tumors may be performed. Table 4 represents a proposal for stratifying GEP-NETs into three groups for purposes of treatment: (1) tumors that are still localized, (2) tumors with lymph node metastases, and (3) tumors with lymph node and hematogenous metastases.

\section{Conclusions}

The proposed standard procedure for the diagnosis of GEP-NETs is simple and easy to perform. Based on the WHO classifications $[14,18]$ and recently published reviews and guidelines $[1-3,19,20]$, it allows for the correct recognition and prognostic stratification of an individual GEP-NET, in order to assure its adequate treatment. It is hoped that this approach will prove helpful for the standardization of the diagnosis and treatment of GEPNETs.

\section{Acknowledgements}

The Meeting on Mallorca was supported by generous grants to ENETS from Novartis and from IPSEN Beaufour.

\section{List of Participants}

List of Participants of the Consensus Conference on the ENETS Guidelines for the Standard of Care for the Diagnosis and Treatment of Neuroendocrine Tumors, Held in Palma de Mallorca (Spain), November 28 to December 1, 2007

Göran Akerström, Department of Surgery, University Hospital, Uppsala (Sweden); Bruno Annibale, University Sapienza Roma, Rome (Italy); Rudolf Arnold, Department of Internal Medicine, Philipps University, Munich (Germany); Emilio Bajetta, Medical Oncology Unit B, Istituto Nazionale Tumori, Milan (Italy); Jaroslava Barkmanova, Department of Oncology, University Hospital, Prague (Czech Republic); Yuan-Jia Chen, Department of Gastroenterology, Peking Union Medical College Hospital, Chinese Academy of Medical Sciences, Beijing (China); Frederico Costa, Hospital Sirio Libanes, Centro de Oncologia, São Paulo (Brazil); Joseph Davar, Department of Cardiology, Royal Free Hospital, London (UK); Wouter de Herder, Department of Internal Medicine, Section of Endocrinology, Erasmus MC, Rotterdam (The Netherlands); Gianfranco Delle Fave, Ospedale S. Andrea, Rome (Italy); Barbro Eriksson, Medical Department, Endocrine Unit, University Hospital, Uppsala (Sweden); Massimo Falconi, Medicine and Surgery, University of Verona, Verona (Italy); Diego Ferone, Departments of Internal Medicine and Endocrinological and Metabolic Sciences, University of Genoa, Genoa (Italy); David Gross, Department of Endocrinology and Metabolism, Hadassah University Hospital, Jerusalem (Israel); Ashley Grossman, St. Bartholomew's Hospital, London (UK); Björn Gustafsson, Medisinsk avd, Gastroseksjon, St Olavs Hospital, Trondheim (Norway); Rudolf Hyrdel, II. Internal Medical Department, University Hospital Martin, Martin (Slovakia); Diana Ivan, Endocrinology and Diabetology, Klinikum der Philipps-Universität, Marburg (Germany); Gregory Kaltsas, G. Genimatas Hospital, Athens (Greece); Reza Kianmanesh, UFR Bichat-Beaujon-Louis Mourier, Service de Chirur- 
gie Digestive, Hôpital Louis Mourier, Colombes (France); UlrichPeter Knigge, Department of Surgery, Rigshospitalet, Copenhagen (Denmark); Beata Kos-Kudła, Slaska Akademia Medyczna Klinika Endokrynologii, Zabrze (Poland); Dik Kwekkeboom, Department of Nuclear Medicine, Erasmus University Medical Center, Rotterdam (The Netherlands); Rachida Lebtahi, Nuclear Medicine Department, Bichat Hospital, Paris (France); Val Lewington, Royal Marsden, NHS Foundation Trust, Sutton (UK); Emmanuel Mitry, Hepatogastroenterology and Digestive Oncology, Hôpital Ambroise-Paré, Boulogne (France); Kjell Öberg, Department of Internal Medicine, Endocrine Unit, University Hospital, Uppsala (Sweden); Juan O’Connor, Instituto Alexander Fleming, Buenos Aires (Argentina); Dermot O'Toole, Department of Gastroenterology and Clinical Medicine, St. James's Hospital and Trinity College Dublin, Dublin (Ireland); UlrichFrank Pape, Department of Internal Medicine, Division of Hepatology and Gastroenterology, Campus Virchow-Klinikum, Charité-Universitätsmedizin Berlin, Berlin (Germany); Mari- anne Pavel, Department of Hepatology and Gastroenterology, Campus Virchow-Klinikum, Charité-Universitätsmedizin Berlin, Berlin (Germany); Aurel Perren, Institut für Allgemeine Pathologie und Pathologische Anatomie der Technischen Universität München, Klinikum r.d. Isar, Munich (Germany); Marco Platania, Istituto Nazionale dei Tumori di Milano, Milan (Italy); Guido Rindi, Department of Pathology and Laboratory Medicine, Università degli Studi, Parma (Italy); Philippe Ruszniewski, Service de Gastroentérologie, Hôpital Beaujon, Clichy (France); Ramon Salazar, Institut Català d'Oncologia, Barcelona (Spain); Klemens Scheidhauer, Klinikum rechts der Isar, TU München, Munich (Germany); Anders Sundin, Department of Radiology, Uppsala University Hospital, Uppsala (Sweden); Waldemar Szpak, Westville Hospital, Mayville (South Africa); Babs Taal, Netherlands Cancer Centre, Amsterdam (The Netherlands); Pavel Vitek, Institute of Radiation Oncology, University Hospital, Prague (Czech Republic); Marie-Pierre Vullierme, Service de Gastroentérologie, Hôpital Beaujon, Clichy (France).

\section{References}

$>1$ Rindi G, Klöppel G, Ahlman H, et al: TNM staging of foregut (neuro)endocrine tumors: a consensus proposal including a grading system. Virchows Arch 2006;449:395-401.

-2 Rindi G, Klöppel G, Couvelard A, et al: TNM staging of midgut and hindgut (neuro) endocrine tumors: a consensus proposal including a grading system. Virchows Arch 2007;451:757-762.

-3 Klöppel G, Rindi G, Anlauf M, Perren A, Komminoth P: Site-specific biology and pathology of gastroenteropancreatic neuroendocrine tumors. Virchows Arch 2007; 451(suppl 1):S9-S27.

4 Plöckinger U, Wiedenmann B: Treatment of gastroenteropancreatic neuroendocrine tumors. Virchows Arch 2007;451(suppl 1):S71S80.

5 Eriksson B, Kloppel G, et al: Consensus guidelines for the management of patients with digestive neuroendocrine tumors well-differentiated jejunal-ileal tumor/carcinoma. Neuroendocrinology 2008;87:8-19.

-6 Jensen RT, Rindi G, et al: Well-differentiated duodenal tumor/carcinoma (excluding gastrinomas). Neuroendocrinology 2006;84: 165-172.

7 Nilsson O, Van Cutsem E, et al: Poorly differentiated carcinomas of the foregut (gastric, duodenal and pancreatic). Neuroendocrinology 2006;84:212-215.
8 O'Toole D, Salazar R, et al: Rare functioning pancreatic endocrine tumors. Neuroendocrinology 2006;84:189-195.

$\checkmark 9$ Rindi G, de Herder WW, et al: Consensus guidelines for the management of patients with digestive neuroendocrine tumors: why such guidelines and how we went about it. Neuroendocrinology 2006;84:155-157.

10 Rindi G, de Herder WW, et al: Consensus guidelines for the management of patients with digestive neuroendocrine tumors: the second event and some final considerations. Neuroendocrinology 2008;87:5-7.

11 Ruszniewski P, Delle Fave G, et al: Well-differentiated gastric tumors/carcinomas. Neuroendocrinology 2006;84:158-164.

12 Lloyd RV: Practical markers used in the diagnosis of neuroendocrine tumors. Endocr Pathol 2003; 14:293-301.

13 Bussolati G, Volante M, Papotti M: Classic and recent special stains used in differential diagnosis of endocrine tumors. Endocr Pathol 2001;12:379-387.

14 Solcia E, Klöppel G, Sobin LH, in collaboration with 9 pathologists from 4 countries: Histological typing of endocrine tumours, ed 2. WHO International Histological Classification of Tumours. Berlin, Springer, 2000 .
15 Pelosi G, Bresaola E, Bogina G, et al: Endocrine tumors of the pancreas: Ki-67 immunoreactivity on paraffin sections is an independent predictor for malignancy: a comparative study with proliferating-cell nuclear antigen and progesterone receptor protein immunostaining, mitotic index, and other clinicopathologic variables. Hum Pathol 1996;27:1124-1134.

16 Reubi JC: Peptide receptor expression in GEP-NET. Virchows Arch 2007;451(suppl 1): S47-S50.

17 Volante M, Righi L, Asioli S, Bussolati G, Papotti M: Goblet cell carcinoids and other mixed neuroendocrine/non-neuroendocrine neoplasms. Virchows Arch 2007;451(suppl 1):S61-S69.

18 DeLellis RA, Lloyd RV, Heitz PU, Eng C: Pathology and Genetics of Tumours of Endocrine Organs. WHO Classification of Tumours. Lyon, IARC Press, 2004.

19 Rindi G, de Herder WW, O’Toole D, Wiedenmann B: Consensus guidelines for the management of patients with digestive neuroendocrine tumors: the second event and some final considerations. Neuroendocrinology 2008;87:5-7.

20 Klöppel G: Tumour biology and histopathology of neuroendocrine tumours. Best Pract Res Clin Endocrinol Metab 2007;21:15-31. 


\section{Erratum}

In the paper by Klöppel et al. [Neuroendocrinology 2009;90:162-166], please replace table 2 with the table below as the Ki67 index value for Grade 1 was incorrect.

Table 2. Grading proposal for GEP-NETs from Rindi et al. [1]

\begin{tabular}{lll}
\hline Grade & Mitotic count, $10 \mathrm{HPF}^{1}$ & Ki67 index, $\%^{2}$ \\
\hline G1 & 1 & $\leq 2$ \\
G2 & $2-20$ & $3-20$ \\
G3 & $>20$ & $>20$
\end{tabular}

${ }^{1} \mathrm{HPF}=$ high-power field $=2 \mathrm{~mm}^{2}$, at least 40 fields evaluated in areas at highest mitotic density.

${ }^{2}$ MIB1 antibody; \% of 2,000 cells in areas of highest nuclear labeling. 